\title{
Vaginal cytokine profile and microbiota before and after lubricant use compared with condomless vaginal sex: a preliminary observational study
}

Susan Tuddenham ${ }^{1 \dagger}$, Christina A. Stennett ${ }^{2 \dagger}$, Richard A. Cone ${ }^{3}$, Jacques Ravel ${ }^{2}$, Andrew N. Macintyre ${ }^{4}$ Khalil G. Ghanem ${ }^{1}$, Xin He $\mathrm{H}^{5}$ and Rebecca M. Brotman ${ }^{2^{*}}$

\begin{abstract}
Background: Limited data suggest that personal lubricants may damage the vaginal mucosal epithelium, alter the vaginal microbiota, and increase inflammation. We compared vaginal cytokine profiles and microbiota before and after vaginal lubricant use and condomless vaginal sex.

Methods: Reproductive-age women were recruited to a 10-week observational cohort study and were asked to self-collect vaginal samples and behavioral diaries daily. This nested case-control analysis utilized samples collected before and after self-reported condomless sexual activity with lubricants (22 case participants) and without lubricants (22 control participants). Controls were matched to cases on race/ethnicity. Microbiota composition was characterized by sequencing amplicons of the $16 \mathrm{~S}$ rRNA gene V3-V4 regions. Cytokine concentrations were quantified using a magnetic bead 41-plex panel assay and read using a Bio-Plex 200 array reader. Wilcoxon signed-rank tests were used to assess baseline differences in vaginal cytokines between cases and controls as well as differences pre- and post-exposure. Linear mixed effects models were used to examine differences in relative post-to-pre change in each individual cytokine between matched cases and controls. Similar analyses were conducted for the microbiota data.

Results: Mean age was 29.8 years (SD 6.8), and 63.6\% were African American. There were few statistically significant changes in cytokines or microbiota before and after exposure in cases or controls. In mixed-effects modeling, the mean relative post-to-pre change of cytokines was higher in cases vs. controls for macrophage derived chemokine (MDC) $(p=0.03)$. The microbiota data revealed no significant changes when measured by similarity scores, diversity indexes and descriptive community state types (CST) transition analyses. However, post sexual activity, the mean relative abundance of $L$. crispatus decreased for those who used lubricants (particularly those who were $L$. iners-dominated prior to exposure).
\end{abstract}

Conclusions: Although there were overall few differences in the vaginal microbiota and cytokine profiles of lubricant users and controls before and after condomless vaginal sex, there was a trend toward decreases in relative abundance

\footnotetext{
*Correspondence: rbrotman@som.umaryland.edu

'Susan Tuddenham and Christina A. Stennett contributed equally to the manuscript.

${ }^{2}$ Institute for Genome Sciences, University of Maryland School

of Medicine, Baltimore, MD, USA

Full list of author information is available at the end of the article
}

(C) The Author(s) 2021. Open Access This article is licensed under a Creative Commons Attribution 4.0 International License, which permits use, sharing, adaptation, distribution and reproduction in any medium or format, as long as you give appropriate credit to the original author(s) and the source, provide a link to the Creative Commons licence, and indicate if changes were made. The images or other third party material in this article are included in the article's Creative Commons licence, unless indicated otherwise in a credit line to the material. If material is not included in the article's Creative Commons licence and your intended use is not permitted by statutory regulation or exceeds the permitted use, you will need to obtain permission directly from the copyright holder. To view a copy of this licence, visit http://creativecommons.org/licenses/by/4.0/. The Creative Commons Public Domain Dedication waiver (http://creativeco mmons.org/publicdomain/zero/1.0/) applies to the data made available in this article, unless otherwise stated in a credit line to the data. 
of L. crispatus following use of lubricant. Future larger studies that take into account osmolarity and composition of lubricants may provide additional insights.

Keywords: Vaginal lubricants, Immunology, Bacterial vaginosis, Sexually transmitted infections

\section{Background}

Over $60 \%$ of American women report use of personal lubricants during intimate sexual activities [1]. Vaginal lubricants are also often administered to improve comfort during clinical gynecological exams. As most of these products are classified by the Food and Drug Administration (FDA) as cosmetics, human safety data is not required prior to commercialization. The FDA classifies lubricants for clinical use as class II medical devices, a category that may pose moderate risk to high to patients [2]. Prior work has linked vaginal products containing nonoxynol-9 (a spermicide) to increased inflammation and risk of HIV acquisition in women [3]. Of concern, non-spermicide containing lubricants have been linked to enhanced vaginal susceptibility to sexually transmitted infections (STIs) in animal models [4] and to an increased risk of rectal STIs in men who have sex with men [5].

The mechanisms for this enhanced risk with non-spermicide containing lubricants are unclear, but may relate to mucosal epithelial damage and increased inflammation as well as to changes in local bacterial populations, because lubricants are often formulated with anti-microbial preservatives such as chlorhexidine [6, 7]. Studies have suggested that some lubricants may inhibit the growth of Lactobacillus species, an essential component of an optimal vaginal microbiota, thus promoting vaginal dysbiosis [6, 8-11]. This may be important as lack of vaginal lactobacilli, in particular lack of Lactobacillus crispatus dominance, has been shown to be associated with significant increase in susceptibility to STIs [12] and HIV acquisition [13]. Additionally, many lubricants are formulated with high concentrations of substances such as glycerol or propylene glycol [14]. These concentrated humectants make them feel comfortably warm when applied to the skin, but also make them markedly hypertonic to mucosal epithelia [6, 14]. Many commercially available lubricants have osmolalities above the World Health Organization (WHO) recommended limit of $1200 \mathrm{mOsm} / \mathrm{kg}[15,16]$. Multiple studies have shown that hypertonic lubricants may be toxic to mucosal epithelial cells, inducing significant epithelial cell shedding in in-vitro and animal models as well as in humans $[6,7$, $17,18]$. For example, one study showed that application of a lubricant with an elevated osmolality caused shedding of large sheets of epithelial cells from rectal tissue [7]. An in vitro study using a three dimensional model of the human vaginal epithelium demonstrated that high osmolality lubricants markedly reduced epithelial barrier properties and induced damage to tissue structures [19].

Elevations in levels of specific "pro-inflammatory" vaginal cytokines have been associated with BV, STIs and enhanced acquisition of HIV [13, 20-22]. Several studies have investigated the impact of vaginal microbicide gels or candidate carrier lubricants on cytokines in the human vagina or upper reproductive tract as potential markers of inflammation, irritation and therefore enhanced HIV acquisition [23-25]. However, none have examined the impact of routine use of vaginal lubricants in women who had condomless vaginal intercourse as compared to condomless vaginal intercourse without lubricant use on both vaginal cytokines and microbiota in a methodical fashion. Despite widespread use, the impact of commercially available vaginal lubricants on the vaginal microenvironment, inflammation and immunity is understudied. Based on our current knowledge, we hypothesized that we would observe a larger increase in (1) pro-inflammatory vaginal cytokines and chemokines and (2) strict and facultative anaerobic bacteria in women reporting use of personal vaginal lubricants (purchased over the counter) during condomless vaginal sex (cases) as compared with women engaging in condomless vaginal sex without lubricant use (controls). In order to test this hypothesis, herein, we compared women's vaginal cytokine and microbiota profiles before and after condomless vaginal sex with or without vaginal lubricant use.

\section{Methods}

This is a nested case-control study utilizing existing samples from a parent longitudinal study conducted between 2009 and 2010 at the University of Alabama at Birmingham (UAB) [26]. For the parent observational cohort study, HIV-negative, reproductive-age, non-pregnant, cis-gender women were recruited and followed over 10 weeks. All women underwent a pelvic examination and microscopy prior to enrollment to evaluate for symptomatic bacterial vaginosis, vaginal candidiasis, or trichomoniasis. Additionally, an endocervical swab was sent for nucleic acid amplification testing for gonorrhea and chlamydia. If a diagnosis of a STI or symptomatic condition was made at baseline, patients were offered treatment but excluded from the study. Participants were offered the opportunity to return to attempt enrollment again 30 days after treatment was completed. Each day, participants self-collected mid-vaginal swabs and filled 
out behavioral diaries (yes/no fields). Dry swabs used for cytokine analyses were placed in empty tubes, and swabs used for microbiota analyses were placed in tubes containing Amies transport medium. All swabs were promptly frozen in participants' home freezers before being returned to the research clinic in weekly batches on frozen ice packs. Once returned, tubes were transferred to long-term storage in $-80^{\circ} \mathrm{C}$ freezers. Samples for cytokine quantification underwent a total of two freeze thaw cycles (one for aliquoting and a final thaw for analysis). All methods were carried out in accordance with relevant guidelines and regulations.

For this analysis, we first selected vaginal samples collected before and after self-reported lubricant use with condomless vaginal intercourse (cases). For comparison, we then chose vaginal samples from race-matched women collected before and after self-reported condomless vaginal intercourse without report of lubricant use (controls) (Fig. 1A, B). For the majority of participants, an individual exposure day (i.e., one day with reported lubricant use or condomless vaginal sex) was identified, and samples collected on the days immediately before and after this exposure were selected (after confirming these days were exposure-free). For participants with consecutive exposure days, the next appropriate day after exposure ended was selected.

\section{Cytokine characterization}

Prior to analysis, frozen, dry vaginal swabs were eluted into $1 \mathrm{~mL}$ of PBS. The eluates were then aliquoted and stored at $-80^{\circ} \mathrm{C}$ until immediately before analysis. Cytokine concentrations in undiluted eluates were quantified in duplicate using a magnetic bead 41-plex panel assay (Millipore HCYTMAG60PMX41BK) performed according to the manufacturer's recommended protocol and read using a Bio-Plex 200 array reader (Bio-Rad). Data were analyzed using Bio-Plex manager software (Bio-Rad). All samples were assayed in a single batch and manufacturer-provided internal controls were included on each assay plate. The 41 measured cytokines and chemokines included: interferon alpha (INF- $\alpha$ ), interferon gamma (INF- $\gamma$ ), interleukin 1 alpha (IL-1 $\alpha$ ), interleukin 1 beta (IL-1 $\beta)$, interleukin 1 receptor antagonist (IL-1RA), interleukin 2 (IL-2), interleukin 3 (IL-3), interleukin 4 (IL-4), interleukin 5 (IL-5), interleukin 6 (IL-6), interleukin 7 (IL-7), interleukin 8 (IL-8), interleukin 9 (IL-9), interleukin 10 (IL-10), interleukin 12 heterodimer p40 and p35 (IL-12p70), interleukin 12 homodimer p40 (IL-12p40), interleukin 13 (IL-13), interleukin 15 (IL15), interleukin 17 alpha (IL-17 $\alpha$ ), tumor necrosis factor alpha (TNF- $\alpha$ ), epidermal growth factor (EGF), fibroblast growth factor (FGF), granulocyte colony stimulating factor (GCSF), granulocyte-macrophage colony-stimulating factor (GM-CSF), monocyte chemotactic protein 1 (MCP-1), interferon gamma-induced protein 10 (IP-10), macrophage Inflammatory protein 1 alpha (MIP-1 $\alpha)$, macrophage inflammatory protein 1 beta (MIP-1 $\beta$ ), tumor necrosis factor beta (TNF- $\beta$ ), vascular endothelial growth factor (VEGF), FMS-like tyrosine kinase 3 ligand (Flt-3L), fractalkine, growth related protein (GRO), monocyte chemotactic protein-3 (MCP-3), macrophage derived chemokine (MDC), platelet derived growth factor $\mathrm{AA}$ dimer (PDGFa), platelet derived growth factor $\mathrm{AB}$ and BBdimer (PDGFab), soluble CD40 ligand (sCD40L), RANTES (CCL5), eotaxin, and transforming growth factor alpha (TGF- $\alpha)$.

\section{Vaginal microbiota characterization}

Vaginal swabs that had been stored in Amies liquid transport medium at $-80^{\circ} \mathrm{C}$ were selected, thawed, and prepared for genomic DNA extraction. The validated procedures employed have been previously published [26]. In brief, enzymatic and physical lysis of bacterial cells was followed by purification of genomic DNA using a QIAsymphony robotic platform and QIAGEN CellFree 500 kits (QIAGEN, Valencia, CA, USA) according to the manufacturer's protocol. This procedure provided between 2.5 and $5 \mu \mathrm{g}$ of high-quality genomic DNA from $300 \mu \mathrm{l}$ of each sample swab stored in Amies transport. The V3-V4 hypervariable regions of the 16S rRNA genes were targeted from genomic DNA using bacterial primers $338 \mathrm{~F}$ and $806 \mathrm{R}$ and were amplified by a two-step polymerase chain reaction (PCR) method [27]. Amplicon pooling, sequencing on an Illumina HiSeq 2500 instrument using a modified PE300 protocol, sequence data pre-processing, and taxonomic assignments were conducted as described by Holm et al. [28]. Vaginal microbiota were assigned community state types (CSTs) [29] using VALENCIA (https://github.com/ravel-lab/VALEN CIA), a nearest-centroid-based algorithm [30]. Four CSTs indicated dominance by Lactobacillus $-L$. crispatus (CST I), L. gasseri (CST II), L. iners (CST III), or L. jensenii (CST V). CST IV, a low-Lactobacillus state, was divided based on the presence and relative abundance of anaerobic organisms-BVAB1 and Gardnerella vaginalis (CST IV-A), G. vaginalis and Atopobium vaginae (CST IV-B), Prevotella spp. and others (CST IV-C) [30, 31].

\section{Statistical analyses}

In order to compare baseline behavioral characteristics between matched cases and controls, asymptotic symmetry and marginal homogeneity tests were used for categorical variables, McNemar's chi-square tests for binary variables, and paired $t$-tests or Wilcoxon signedrank tests for continuous variables. For samples with vaginal cytokine measurements below the lower limit 


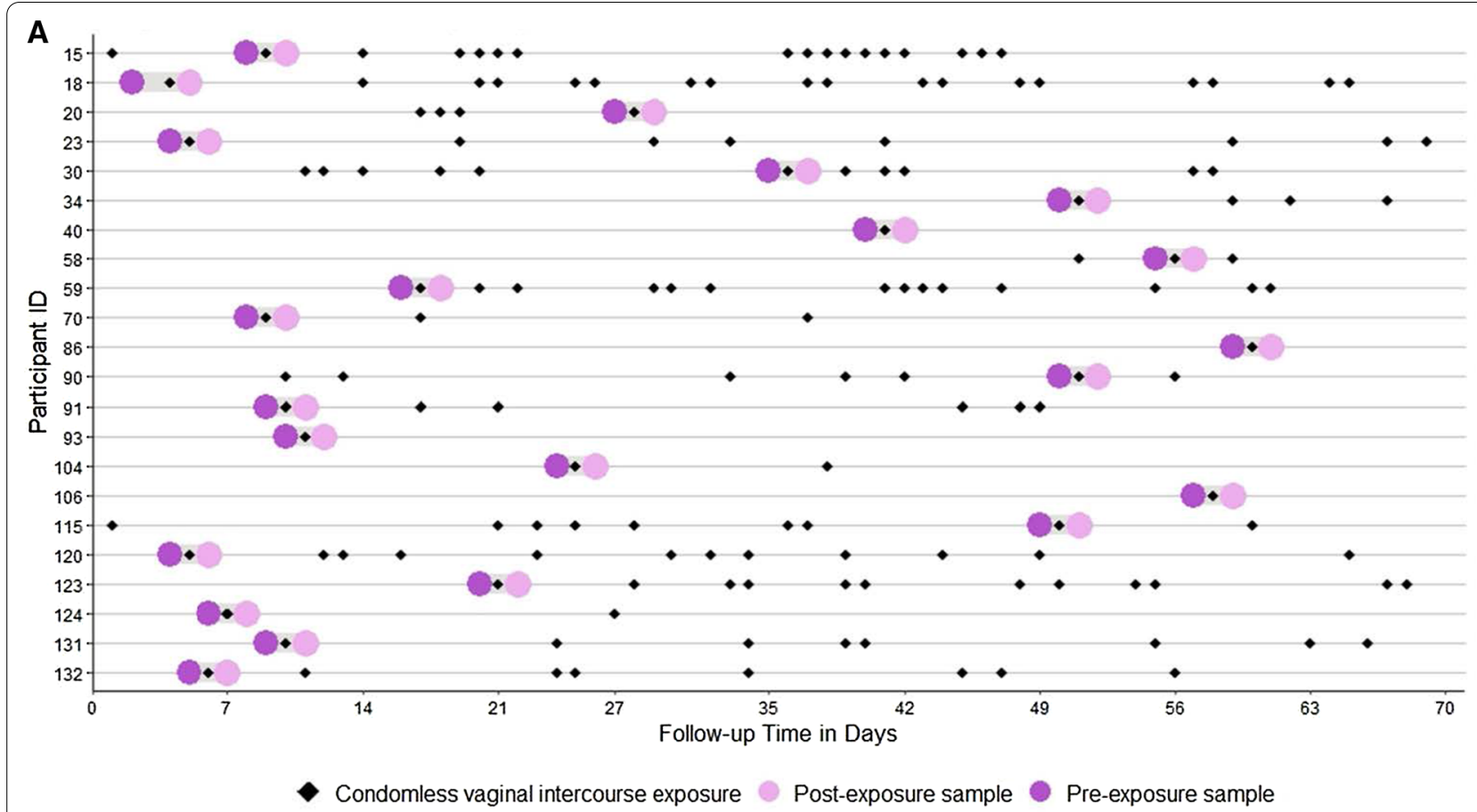

B

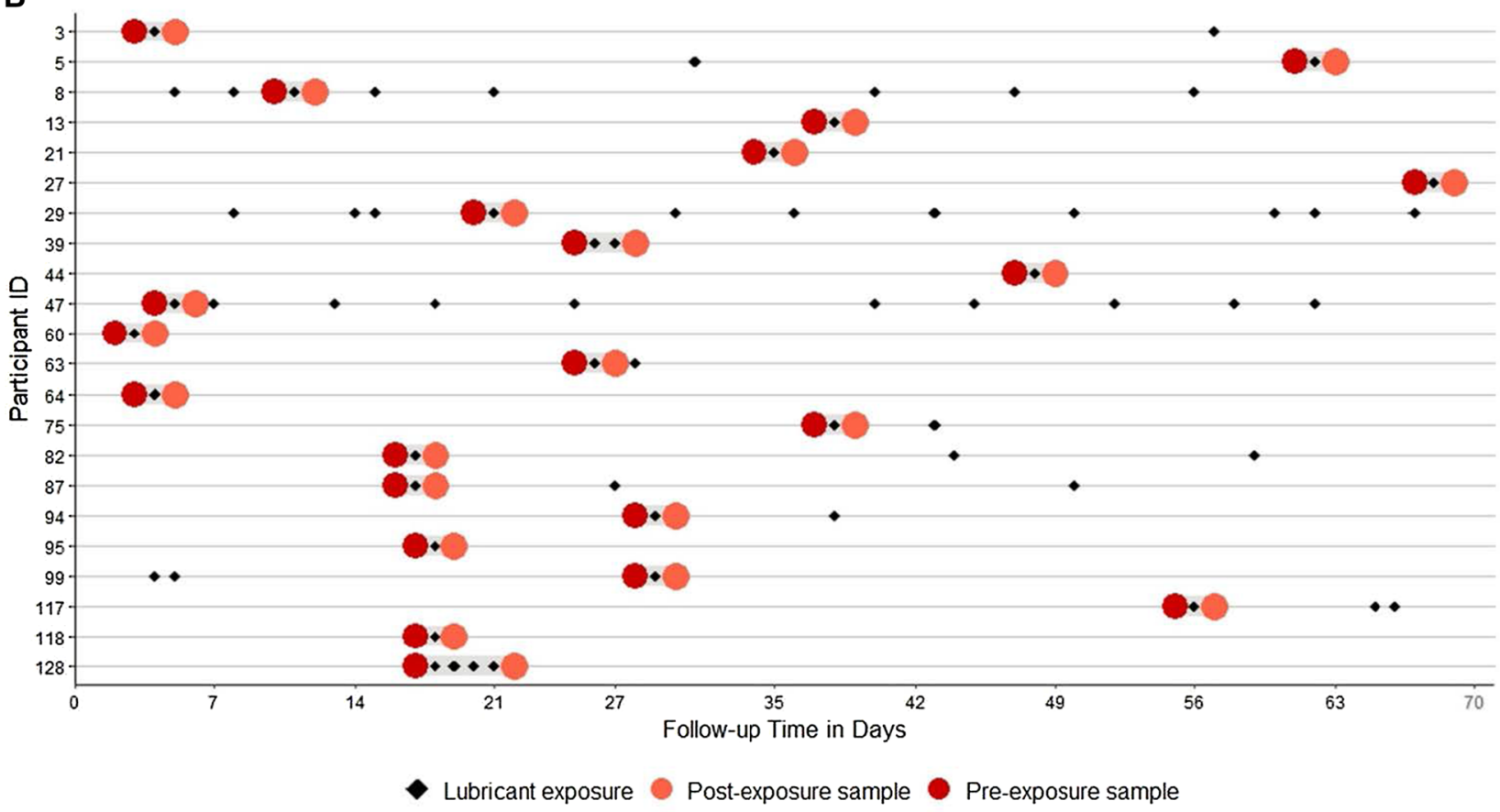

Fig. 1 A Longitudinal plot for controls $(n=22)$ including instances of self-reported condomless vaginal sex without lubricant use and pre- and post-exposure samples selected for analysis. B Longitudinal plot for cases $(n=22)$ including instances of self-reported condomless vaginal sex with lubricant use and pre- and post-exposure samples selected for analysis

of detection, a number halfway between 0 and the lowest measured value for that particular cytokine for that particular plate was imputed [32]. Cytokines that were below the level of quantitation in $>90 \%$ of all samples (IL2, IL-3, IL-5, IL-9, IL-10, IL-15, TNF- $\beta$ ) were excluded from the analysis. One analyte, IL-1RA, was above 
the upper limit of quantitation in $96 \%$ of samples and excluded from the analysis. In seven samples one analyte other than IL-1RA was above the upper limit. These seven values were imputed as $10,000 \mathrm{pg} / \mathrm{mL}$, the upper limit of quantitation for the assay. Wilcoxon signed-rank tests were used to assess baseline differences in vaginal cytokine levels between matched cases and controls and differences in cytokines between pre- and post-exposure samples. We assessed differences in pre-exposure sample CSTs between cases and controls with the marginal homogeneity test. The Shannon Diversity Index (SDI) was calculated to measure the diversity of bacterial species within each vaginal sample [33]. The pre-exposure sample SDI was subtracted from the post-exposure value; a positive change in SDI indicated greater bacterial species diversity in the post-exposure sample. We then compared the median SDI change values between cases and controls using the Wilcoxon signed-rank test. In addition, we assessed the Yue Clayton theta distance [34], which measured the similarity in bacterial communities between pre- and post-exposure samples, and compared the theta distances for cases and controls using the Wilcoxon signed-rank test.

Linear mixed-effects and generalized linear mixedeffects models were used to assess differences in individual cytokines before and after exposure, and by case-control status. In the linear mixed-effects models for the cytokine analysis, the outcome was the $\log _{10}$-transformed ratio of the post-exposure cytokine level and the pre-exposure cytokine level (i.e., the relative post-to-pre change), and a normally distributed random effect term was used to adjust for matched pairs. For the vaginal microbiota analysis, to assess post-to-pre differences in the relative abundance of specific bacterial taxa, we employed similar modeling methods. Linear mixed-effects models were used to assess differences in the most abundant bacterial taxa before and after exposure by case-control status. To calculate the post-to-pre relative abundance ratio outcome for each bacterial taxa, we added a constant (1) to the relative abundances in all samples and divided the post-exposure relative abundance value by the pre-exposure value to obtain a ratio (Formula 1), which was then $\log _{10}$-transformed. A normally distributed random effect term was included in the model to adjust for matched pairs.

Formula 1:

$$
\text { Ratio }=\frac{(" P o s t " \text { sample relative abundance })+1}{(" P r e " \text { sample relative abundance })+1}
$$

Principal component analysis (PCA) was also conducted to visualize the pre- to post-exposure cytokine ratio in cases versus controls. Following an approach presented by Arnold et al. [35], we defined a binary measurement for "inflammation" as having at least three out of seven predetermined inflammatory cytokines (IL-1 $\alpha$, IL-1 $\beta$, IL-8, MIP- $1 \alpha$, MIP- $1 \beta$, IP-10, and RANTES) in the upper quartile. MIP- $1 \alpha$ and IP-10 were added to the list of inflammatory cytokines based on relevant data from Masson et al. [36] showing a strong association with HIV seroconversion. A logistic mixed-effects model adjusting for matched pairs and within-subject correlations between pre- and post-exposure measures was applied with the binary inflammation score as the outcome. All analyses were conducted using STATA v14 (Stata Corp, College Station, TX) and R. PCA plots were constructed utilizing SIMCA 15 (Umetrics AB, Umea, Sweden).

\section{Results}

Paired pre- and post-exposure samples from 22 lubricant users and 22 race-matched controls were analyzed for a total of 88 samples. $63.6 \%$ of participants were African American, and the mean age was 30 years (SD 6.8) (Table 1). There were no statistically significant differences between cases and controls in terms of age, douching, hormonal contraception (HC) use, or number of sexual partners, though cases were more likely to report lubricant use in the prior 6 months as compared to controls $(\mathrm{p}=0.03)$ (Table 1, also see Additional file 2: Table S5 for data). Although cases reported more condomless vaginal sex compared to cases (average reporting of 3 days versus 7 days, respectively), the difference was not statistically significant ( $p=0.25$; Fig. $1 \mathrm{~A}, \mathrm{~B})$.

\section{Cytokine analysis}

Prior to exposure, the median eotaxin, Flt-3L, and PDGFab were significantly higher in cases as compared to controls (Additional file 1: Table S1) with a similar trend in VEGF. Among controls, Wilcoxon signed-rank tests indicated that when comparing cytokines pre- to postexposure as continuous measures, the median MCP-3 and $\mathrm{SCD} 40 \mathrm{~L}$ levels were significantly higher before exposure (Additional file 1: Table S2), with a similar trend in IL1-7 $\alpha$. There were no significant differences when comparing median cytokines post- to pre-exposure within cases (lubricant users), though there was a trend towards higher median eotaxin pre-exposure $(\mathrm{p}=0.05)$ (Additional file 1: Table S3).

In mixed-effects modeling, adjusting for log-transformed baseline cytokine level, the mean log-transformed relative pre- to post-exposure change of cytokines was higher in cases as compared to controls for MDC $(\mathrm{p}=0.03)$, (Additional file 1: Table S4). A PCA plot of the pre- to post-exposure cytokine ratios (Fig. 2) showed no clear grouping using the first two principal components by case-control status. Sample 8 had a higher value in the 
Table 1 Demographic and behavioral characteristics

\begin{tabular}{|c|c|c|c|}
\hline & $\begin{array}{l}\text { Overall } \\
\mathrm{N}=44\end{array}$ & $\begin{array}{l}\text { Controls } \\
n=22\end{array}$ & $\begin{array}{l}\text { Lubricant } \\
\text { users } n=22\end{array}$ \\
\hline Age, Mean (SD) & $29.8(6.8)$ & $30.5(7.0)$ & $29.1(6.8)$ \\
\hline \multicolumn{4}{|l|}{ Race } \\
\hline African American & $28(63.6)$ & $14(63.6)$ & $14(63.6)$ \\
\hline White & $14(31.8)$ & $7(31.8)$ & $7(31.8)$ \\
\hline Latina & $2(4.6)$ & $1(4.6)$ & $1(4.6)$ \\
\hline \multicolumn{4}{|l|}{ Douching frequency last 60 days } \\
\hline None & $32(72.7)$ & $15(68.2)$ & $17(77.3)$ \\
\hline Monthly & $3(6.8)$ & $3(13.6)$ & $0(0.0)$ \\
\hline Every now and then & $3(6.8)$ & $1(4.6)$ & $2(9.1)$ \\
\hline No answer & $6(13.6)$ & $3(13.6)$ & $3(13.6)$ \\
\hline Vaginal Lubricant use last 60 days $(Y)^{*}$ & $11(25.6)$ & $2(9.1)$ & $9(42.9)$ \\
\hline Current $\mathrm{HC}$ use $(\mathrm{Y})$ & $11(25.0)$ & $5(22.7)$ & $6(27.3)$ \\
\hline \multicolumn{4}{|l|}{ Number sex partners last 60 days } \\
\hline 0 & $3(6.8)$ & $2(9.1)$ & $1(4.6)$ \\
\hline 1 & $37(84.1)$ & $19(86.4)$ & $18(81.8)$ \\
\hline 2 & $4(9.1)$ & $1(4.6)$ & $3(13.6)$ \\
\hline \multicolumn{4}{|l|}{ Pre-exposure sample $\mathrm{pH}^{*}$} \\
\hline $4.0-4.5$ & $25(58.1)$ & $12(54.6)$ & $13(61.9)$ \\
\hline $4.6-5.0$ & $10(23.3)$ & $6(27.3)$ & $4(19.1)$ \\
\hline$>5.0$ & $8(18.6)$ & $4(18.2)$ & $4(19.1)$ \\
\hline \multicolumn{4}{|l|}{ Pre-exposure sample Nugent category } \\
\hline No BV & $23(52.3)$ & $13(59.1)$ & $10(45.5)$ \\
\hline Intermediate & $8(18.2)$ & $2(9.1)$ & $6(27.7)$ \\
\hline BV & $13(29.6)$ & $7(31.8)$ & $6(27.7)$ \\
\hline \multicolumn{4}{|l|}{ Pre-exposure sample CST** } \\
\hline CST-I, L. crispatus-dominated & $8(18.2)$ & $6(28.6)$ & $2(9.1)$ \\
\hline CST-II, L. gasseri-dominated & $5(11.4)$ & $2(9.5)$ & $3(13.6)$ \\
\hline CST-III, L. iners-dominated & $14(31.8)$ & $5(23.8)$ & $9(40.9)$ \\
\hline CST-IVA, Low Lactobacillus & $7(15.9)$ & $3(14.3)$ & $4(18.2)$ \\
\hline CST-IVB, Low Lactobacillus & $6(13.6)$ & $3(14.3)$ & $3(13.6)$ \\
\hline CST-IVC, Low Lactobacillus & $2(4.5)$ & $1(4.8)$ & $1(4.5)$ \\
\hline CST-V, L.jensenii-dominated & $1(2.3)$ & $1(4.8)$ & $0(0.0)$ \\
\hline \multicolumn{4}{|l|}{ Pre-exposure sample CST** } \\
\hline CST-I/IIN, L. crispatus/gasseri/jensenii-dominated & $14(32.6)$ & $9(42.9)$ & $5(22.7)$ \\
\hline CST-III, L. iners-dominated & $14(32.6)$ & $5(23.8)$ & $9(40.9)$ \\
\hline CST-IVA/B/C, Low Lactobacillus & $15(34.9)$ & $7(33.3)$ & $8(36.4)$ \\
\hline
\end{tabular}

HC: hormonal contraception, CST:community state type. Cases were more likely to report vaginal lubricant use in the last 60 days ( $p=0.03)$. There were no statistically significant differences between cases and controls in terms of age, race, douching, hormonal contraception (HC) use, number of sexual partners or pre-exposure $\mathrm{pH}$, Nugent category, or CST $(p>0.05) .{ }^{*} 1$ case missing data. ${ }^{* *} 1$ control missing data

first principal component, while sample 21 had a higher value in the second principal component. These differences do not appear to be driven by any single cytokine.

Using a logistic mixed-effects model to analyze the binary inflammation score, there were no statistically significant differences in the odds of having an inflammatory vaginal microenvironment between cases and controls adjusting for whether it was sampled prior to or following exposure, within-subject correlations, and matched pairs $(p=0.16)$. Furthermore, there were no significant differences in the odds of having an inflammatory vaginal microenvironment between cases and controls in the pre-exposure samples after adjusting for matched pairs $(p=0.16)$. Finally, there were no significant differences in the odds of having an inflammatory vaginal microenvironment between pre- and post-exposure measures 


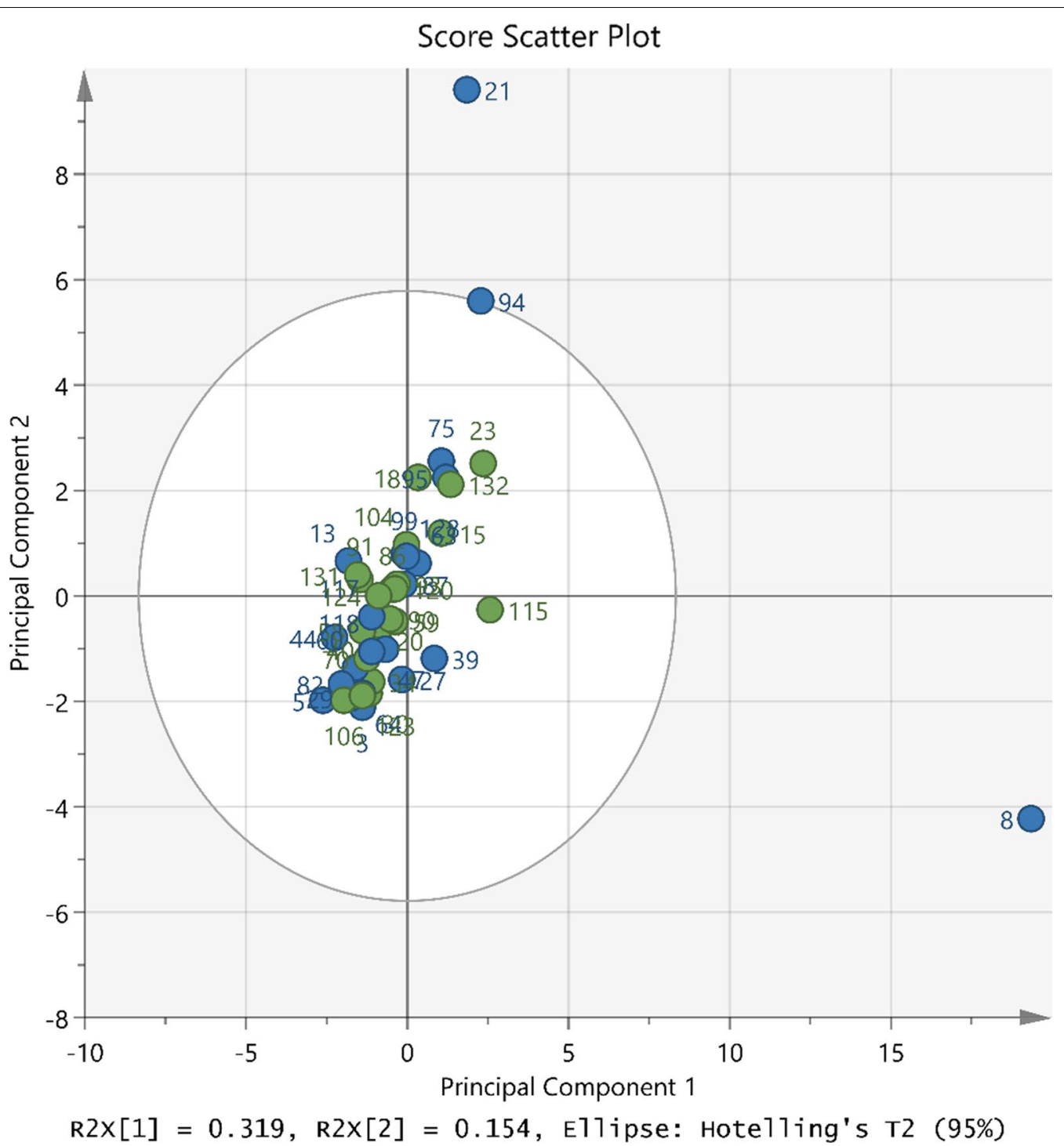

Fig. 2 Principal components analysis of pre- to post-exposure cytokine ratios. Blue circles indicate cases (lubricant users) and green circles indicate controls

within controls $(\mathrm{p}=0.14)$ or cases $(\mathrm{p}>0.99)$ after adjusting for within-subject correlations. Due to the small sample size and exploratory intent of the pilot study, correction for multiple comparisons was not conducted, but none of our findings would have been significant after correction according to the Bonferroni method.

\section{Vaginal microbiota analysis}

The distribution of pre-exposure CSTs between cases and controls was similar whether a 7-CST $(\mathrm{p}=0.56)$ or a 3 -CST scheme $(\mathrm{p}=0.29)$ were used, with the proportion of participants having a low-Lactobacillus sample prior to exposure being nearly equal in both groups (Table 1 ).
The CSTs and bacterial taxa relative abundances of the samples included in the analysis are shown in Fig. 3. There were 3 (13\%) CST-discordant case pairs (pre- and post-exposure samples) and 5 (24\%) CST-discordant control pairs. The pre-to-post CST transitions observed were Lactobacillus-dominated to CST IV-B or CST IV-C (2 cases), CST IV-B to CST III (2 controls), CST IV-A to CST IV-B ( 1 case and 2 controls), and CST V to CST III (1 control).

The median change in SDI was -0.03 and 0.02 for cases and controls, respectively (Additional file 1: Figure S1A). These results suggest slightly higher species diversity in pre-exposure samples for cases and post-exposure 


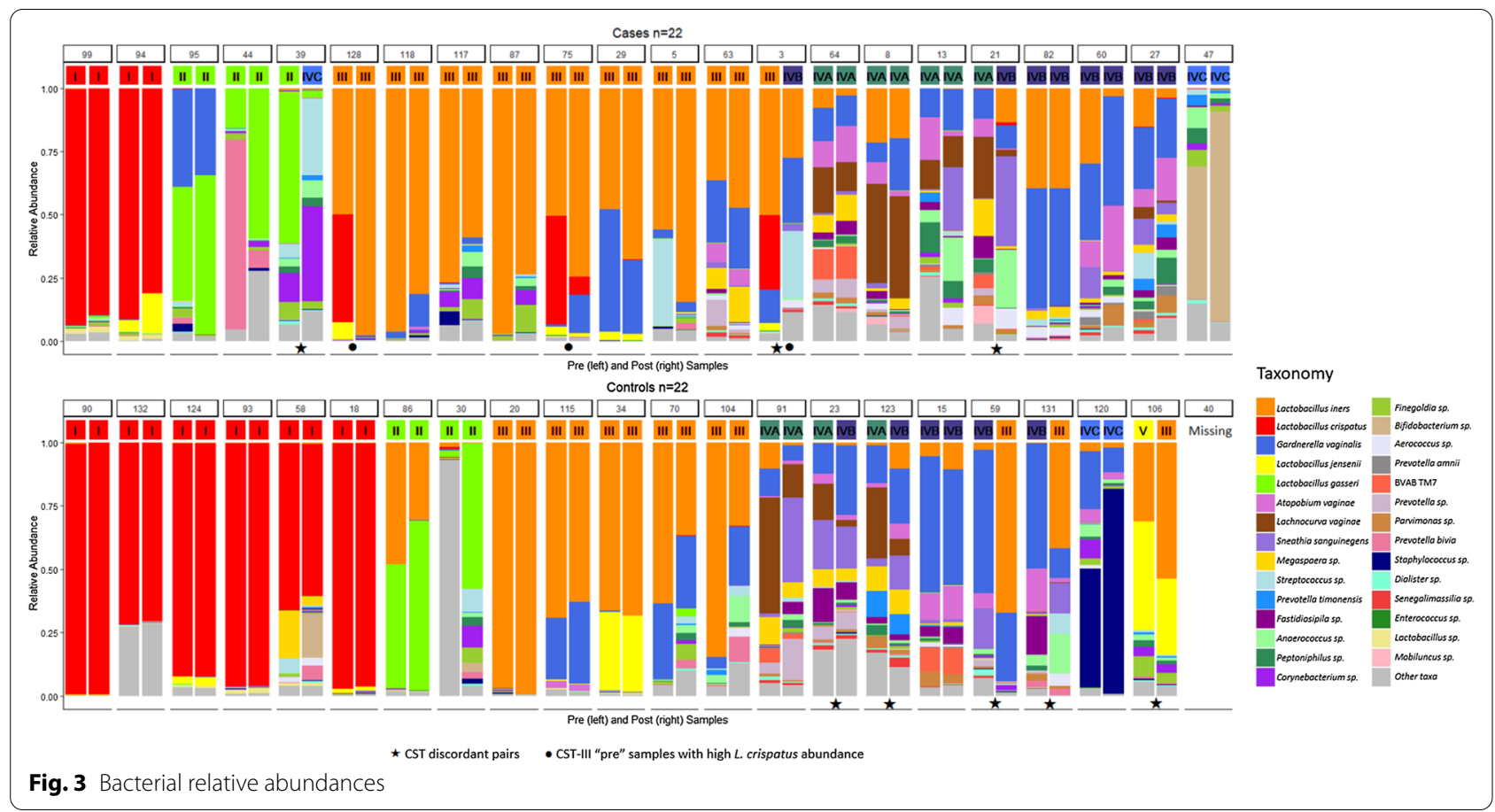

samples for controls; however, the differences by casecontrol status were not statistically significant $(\mathrm{p}=0.24)$. The median Yue Clayton theta distance was 0.72 for cases and 0.65 for controls indicating both had relatively high similarity, and there were no statistically significant differences in post-to-pre bacterial taxa between cases and controls ( $p=0.52$, Additional file 1: Figure S1B).

When focusing on the seven taxa with the highest relative abundance, there were no statistically significant differences between pre-exposure samples comparing cases and controls. The adjusted linear mixed-effects models showed a significant difference in the mean $\log _{10}$-transformed post-to-pre relative abundance $(\mathrm{p}=0.014)$ (Table 2). When controlling for the pre-exposure sample $L$. crispatus relative abundance, the mean post-to-pre $L$. crispatus relative abundance ratio was lower (i.e., mean post-exposure relative abundance was lower than the pre-exposure relative abundance, producing a ratio $<1)$ in cases compared to controls. This finding is demonstrated in the relative abundance bar charts (Fig. 3). Among cases whose pre-exposure sample was classified as $L$. iners-dominated CST III, but also had a sizeable proportion of $L$. crispatus present, $L$. crispatus abundance was significantly lower after lubricant exposure in the post-exposure sample. There were no examples of controls with pre-exposure samples that were

Table 2 Top 7 most abundant taxa in the vaginal microbiota comparing mean post-to-pre-exposure ratio by case or control status

\begin{tabular}{llll}
\hline Taxa & $\begin{array}{l}\text { Controls } \\
\text { Mean post/pre ratio (SD) }\end{array}$ & $\begin{array}{l}\text { Cases } \\
\text { Mean post/pre ratio (SD) }\end{array}$ & $\begin{array}{l}\text { Generalized mixed } \\
\text { effect model p-value }\end{array}$ \\
\hline L. iners & $1.03(0.19)$ & $1.01(0.12)$ & 0.91 \\
L. crispatus & $1.00(0.01)$ & $0.96(0.10)$ & 0.01 \\
Gardnerella vaginalis & $1.00(0.09)$ & $1.02(0.06)$ & 0.10 \\
L.jensenii & $1.00(0.02)$ & $1.00(0.03)$ & 0.97 \\
L. gasseri & $1.03(0.12)$ & $1.01(0.12)$ & 0.46 \\
Atopobium vaginae & $0.99(0.03)$ & $1.00(0.05)$ & 0.40 \\
Candidatus Lachnocurva vaginae (formerly BVAB1) & $0.98(0.06)$ & $0.99(0.04)$ & 0.12 \\
\hline
\end{tabular}

$S D$ standard deviation, $B V A B 1$ bacterial vaginosis associated bacteria 1

between cases and controls only for L. crispatus CST III with high L. crispatus relative abundance, and 
the same trend was not seen in cases or controls with pre-exposure samples that were classified as $L$. crispatusdominated CST I.

\section{Discussion}

Despite our hypothesis that we would observe a larger increase in pro-inflammatory cytokines and chemokines after lubricant use in this observational cohort, there were few statistically significant differences in vaginal cytokine profiles before and after exposure in lubricant users versus controls. For the three cytokines (Flt-3L, PDGFab, and eotaxin) which were statistically significantly different between the two groups at baseline, there was no statistically significant pre- to post-exposure change between groups in multivariate modeling. The post- to pre-exposure change of only one chemokine (MDC) was statistically significantly different between groups in multivariate modeling. MDC is a chemokine that recognizes the CCR4 receptor, is selective for Th2 cells, and expression has been shown to be induced by IL-4 and IL-13 [37-39]. MDC is one of seven cytokines that was found in a LASSO cytokine model to classify women as STI/BV positive or negative [21]. However, the lack of differences in MDC comparing pre- and postexposure samples in lubricant users and the lack of differential change in other inflammatory cytokines or chemokines in lubricant users versus controls makes it difficult to interpret this isolated result. Certainly, in this cohort, there is no clear pattern to suggest a markedly pro-inflammatory vaginal microenvironment induced by lubricant use. Some previous studies (primarily from in vitro models) had raised concerns regarding the ability of lubricants (especially hyperosmolar lubricants) to lead to mucosal irritation and epithelial cell damage $[6,7,9,17,40]$. If true, this presumably would lead to a pro-inflammatory vaginal cytokine profile with potential implications for women's reproductive health, including elevated risk of acquisition of STIs and HIV. While a single study found transcriptional upregulation of inflammatory genes in the upper reproductive tract with the use of a "universal placebo gel" previously thought to be inert [25], the results of our study are supported by results from two other placebo-controlled microbicide studies in which neither placebo vaginal lubricant nor microbicide resulted in increases in cervicovaginal pro-inflammatory cytokines [23, 24].

Other than direct cellular toxicity, concerns have been raised that lubricants could negatively impact the vaginal microbiota, leading to decreases in beneficial lactobacilli $[9,41]$, which in turn might have implications for reproductive health including increased susceptibility to STIs and HIV $[31,42]$. We similarly hypothesized there would be a larger increase in strict and facultative anaerobic bacteria post-exposure in lubricant users compared to controls. We did not detect such a difference. Overall, we found few statistically significant differences at baseline between lubricant users or controls or changes in the overall structure of the vaginal microbiota before and after lubricant use. However, one taxon, $L$. crispatus, had higher relative abundance in pre-lubricant samples compared to post-exposure samples for cases. This is interesting given other studies have found a lack of L. crispatus dominance to be associated with a significant increase in susceptibility to HIV acquisition [13]. This is also noteworthy in light of several recent in vitro studies. The first found that the feminine moisturizer Vagisil inhibited L. crispatus growth [9]. Another found that two lubricant products (Replens Long-Lasting moisturizer and TrimoSan) inhibited the growth of L. crispatus, while another product (Replens Silky Smooth) enhanced growth [41]. A third paper found that lubricants containing chlorhexidine gluconate or nonoxynol-9 significantly inhibited Lactobacillus spp. growth, while other lubricants decreased the attachment of lactobacilli to vaginal epithelial cells in vitro [11]. These results, and ours, point to the need for larger, controlled studies to understand the impact of specific lubricants on the vaginal microbiome.

Animal and in vitro studies have suggested that exposure to semen may elicit the expression of pro-inflammatory cytokines in the vaginal tract $[43,44]$. One study by Sharkey et al. demonstrated upregulation of several cytokine mRNAs in the human cervix, including IL- $1 \alpha$, IL-6, and IL-8, $12 \mathrm{~h}$ after condomless vaginal intercourse compared to $36 \mathrm{~h}$ prior [45]. We did not observe these changes in our analysis of pre- and post-vaginal sex exposures. In fact, of the cytokines or chemokines that were statistically significantly different in pre- and post-exposure samples (MCP-3 and $\mathrm{sCD} 40 \mathrm{~L}$ ), all were lower postvaginal sex than pre-vaginal sex. There could be several reasons for this. First, the area sampled and the analyses were different: the Sharkey study utilized cervical biopsies and looked for mRNA expression; we utilized vaginal swabs and directly measured secreted cytokine concentrations. Secondly, there was more variability in the timing of when our "post" sample was taken in relation to sexual intercourse (generally $12-36 \mathrm{~h}$ after). Furthermore, in the Sharkey study, patients used condoms for 5 days and then completely abstained from sexual intercourse for 2 days before the study was conducted. Our samples were taken from an observational cohort, and we did not require women to abstain from intercourse. It is possible that differences pre- and post-exposure to semen might be blunted in women engaging in regular sexual intercourse. 
A strength of our study is the race-matched pre- to post-exposure design that enabled us to systematically assess real-world vaginal lubricant use in women compared to vaginal intercourse without lubricant, allowing us to assess if any findings were the result of lubricant use versus inflammatory changes following sexual activity/vaginal intercourse. We matched on race as it was strongly associated with lubricant use and CST in the Parent study, and based on prior literature, has been associated with several unmeasured factors (e.g., sexual networks and health behaviors) that may confound this analysis $[46,47]$. We were unable to match on pre-exposure CST due to low sample size. However, future studies may investigate how various vaginal bacterial communities are affected by lubricant exposure, as CSTs may have varying resilience to extrinsic exposures.

Our research group [48, 49] and others [50-53] have validated self-collection of mid-vaginal swabs used for immunologic and microbiota analyses, and the practice is now widely used. The daily at-home sampling allows population-based longitudinal field studies. Our samples underwent two freeze thaw cycles. Several studies have shown low numbers $(\leq 2)$ of freeze thaw cycles have negligible impact on the concentration of most cytokines [54-58]. Additionally, we focused on higher abundant bacterial taxa in this analysis. Data from gut microbiota literature suggests that storage of samples over hours to days at room temperature gives similar results to freezing immediately at $-80^{\circ} \mathrm{C}$, with only very low abundant taxa being affected [59-62]. Given the fact that samples were quickly frozen, we do not anticipate that the storage protocols would have affected the relative abundance of the relevant, highly abundant taxa.

However, there are significant limitations to our study. The daily diary did not collect information on which specific lubricants women used, and therefore, we were unable to assess whether these lubricants had elevated osmolalities or contained anti-microbial components. While there is a range of osmolalities and ingredients across commercially available personal lubricant products, in the period of time that this study was conducted, the osmolalities of commercially available lubricants were generally high [6]. Nonetheless, it is still possible in that women using frequent or large amounts of product, lubricants with elevated osmolalities or lubricants with anti-microbial components might have a different immune response. Secondly, since this was an exploratory analysis with a small sample size, we did not correct for multiple comparisons. None of our findings would have been statistically significant after correction for multiple comparisons. Thirdly, our method of imputation for undetectable cytokines, while widely used in the literature $[32,63]$, does have the potential to over or under-estimate true cytokine levels.

Additionally, we analyzed samples taken from an observational cohort study [26] that was powered for different aims. Small sample size was a limitation. The 22 cases and 22 controls represent a convenience sample from the original cohort, and we were only able to match one control per case on race. Furthermore, we did not mandate that women abstain from either condomless sexual intercourse or vaginal lubricant use prior to sample collection as it was an observational study. At baseline, women reported their vaginal lubricant use in the 60 days prior to enrollment in the study. Nine cases (41\%) and two controls (9\%) reported vaginal lubricant use in that period, including KY-Jelly $(n=7)$, Vaseline $(\mathrm{n}=1)$, Silk $(\mathrm{n}=1)$, mineral oil $(\mathrm{n}=1)$, and an unspecified edible lubricant $(n=1)$. Although we confirmed that controls did not use any vaginal lubricants after enrollment, we were unable to verify whether cases continued use of the same lubricant product on the exposure day of interest. Post- to pre-exposure changes in cytokines might be blunted in women regularly engaging in condomless sexual intercourse or regularly utilizing vaginal lubricants. Again, because this was an observational study based on secondary data, the timing of sampling after intercourse/lubricant use was not rigidly controlled. While most post-exposure samples were taken between 12 and $36 \mathrm{~h}$ after intercourse, in 2 participants, this was substantially longer. It is possible that impacts on vaginal cytokines or microbiota are acute and short-lived (i.e., occurring shortly after use and quickly resolving), which could still lead to increased vulnerability (e.g., to HIV acquisition) but would not have been captured by our sampling methods. In addition, the methods used in this study to describe the vaginal microbiota do not differentiate between dead and live bacteria, and it is unknown how long DNA from dead bacteria can be detected in the vagina. It is possible that bacteria that were killed immediately after exposure would still be detectable in samples collected one day after exposure but not two or three days later. However, we posit that DNA detected from dead bacteria would constitute a small part of the result and not confound the observed associations.

Two participants (\#44, a case, and \#30, a control) had pre-exposure samples that had the highest similarity to the centroid of CST II by the VALENCIA algorithm. These two samples were dominated by Klebsiella and $P$. bivia, respectively but did contain a modest proportion of $L$. gasseri and were consequently placed in the L. gasseri-dominated CST II by the algorithm. In a sensitivity analysis, we reassigned these samples to CST IV-C; however, there was still no association between pre-exposure CST and lubricant use using marginal homogeneity tests. 
We also did not detect a difference in post-to-pre L. gasseri relative abundance between cases and controls.

Finally, we estimated the presence and relative abundance of key vaginal bacterial via $16 \mathrm{~S}$ rRNA gene amplicon sequencing. However, it is possible that lubricant could affect not only the abundance of key bacteria but also their functional output-for example, the production of protective lactic acid from lactobacilli. We did not measure vaginal metabolites in this study. The observed difference in L. crispatus appears to be driven by cases whose pre-exposure samples were $L$. iners-dominated (CST III); however, there were no comparable instances among controls where a pre-exposure sample was $L$. iners-dominated but with a high relative abundance of L. crispatus, so it remains unknown what impact sex in the absence of lubricant use would have on this profile. Behavioral diaries were submitted weekly, and there may have been information bias in accurately reporting or recalling daily lubricant use or vaginal sex. We were not able to verify condomless vaginal sex or lubricant use with any biological measures.

\section{Conclusions}

Although overall there were few differences in the vaginal microbiota and cytokine profiles of lubricant users and controls before and after condomless vaginal sex, there was a trend toward decreases in relative abundance of L. crispatus following use of lubricant. Within the limitations of the observational study design, our results are intriguing, but far from definitive. Larger, prospective, well-controlled studies with higher density sampling to carefully assess the impact of different types of overthe-counter vaginal lubricants, including those with high osmolarity, are needed to further evaluate the effect of vaginal lubricants on the vaginal microenvironment and inflammation.

\section{Supplementary Information}

The online version contains supplementary material available at https://doi. org/10.1186/s12879-021-06512-x.

\footnotetext{
Additional file 1: Table S1. Comparing baseline vaginal cytokines prior to lubricant use with condomless vaginal sex (cases) to baseline cytokine profiles prior to condomless vaginal sex without lubricant use (controls): Wilcoxon signed-rank test. Table S2. Comparing vaginal cytokines before ("pre") and after ("post") condomless vaginal intercourse in $\mathrm{N}=22$ controls: Wilcoxon signed-rank test. Table S3. Comparing vaginal cytokines before ("pre") and after ("post") condomless vaginal intercourse with lubricant in $\mathrm{N}=22$ cases: Wilcoxon signed-rank test. Table S4. Multivariable modeling assessing differences in log-transformed pre-to-post ratio in cases versus controls. Figure S1. A. Change in Shannon diversity of the vaginal microbiota pre-to-post in cases compared to controls. B. Change in Yue Clayton theta distance of the vaginal microbiota pre-to-post in cases compared to controls.
}

Additional file 2: Table S5. Cytokine Concentrations and Metadata.

\section{Acknowledgements}

Multiplex cytokine analysis was performed in the Immunology Unit of the Duke Regional Biocontainment Laboratory (RBL) under the direction of Drs. Andrew Macintyre and Greg Sempowski. The Duke RBL received partial support for construction from the National Institutes of Health, National Institute of Allergy and Infectious Diseases (UC6-Al058607).

\section{Author's contributions}

RB conceived the study. CS selected samples and conducted data cleaning. ST, $\mathrm{CS}$ and $\mathrm{XH}$ conducted the statistical analysis. RB, RC, KG, AM, and JR contributed to data interpretation, drafting and reviewing the paper. All authors read and approved the final manuscript.

\section{Funding}

This study was supported by a National Institute of Allergy and Infectious Diseases (NIAID) grants R01-Al1 19012 and UH2-Al083264. Additionally, ST is supported by NIAID grant K23-Al125715, and CS is supported by National Institute on Aging grant T32-AG000262. The content is solely the responsibility of the authors and does not necessarily represent the official views of the National Institutes of Health.

\section{Availability of data and materials}

The $16 \mathrm{~S}$ rRNA gene sequences and CST assignments will be accessible using the National Center for Biotechnology Information (NCBI) Sequence Read Archive (SRA) BioProject accession number PRJNA208535, https://www.ncbi. nlm.nih.gov/bioproject/PRJNA208535. Cytokine concentration data and other demographic and health behavior metadata are included in Additional file 2: Table S5.

\section{Declarations}

Ethics approval and consent to participate

Informed consent was obtained from every participant during the original (parent) study. IRB approvals were obtained from the University of Alabama Birmingham (\#F090430006), University of Maryland Baltimore (\#HP-00041351), and Johns Hopkins (IRB00136266). All methods were performed in accordance with the relevant guidelines and regulations.

\section{Consent for publication}

Not applicable.

\section{Competing interests}

JR is a co-founder of LUCA Biologics, a biotechnology company focusing on translating microbiome research into live biotherapeutic drugs for women's health. ST has been a consultant for LUCA Biologics, Biofire Diagnostics and Roche Molecular Diagnostics and has received a speaker honorarium from Roche Molecular Diagnostics and Medscape. ST also receives royalties from UPTODATE. All other authors declare no competing interests.

\section{Author details}

'Department of Medicine, Johns Hopkins University School of Medicine, Baltimore, MD, USA. ${ }^{2}$ Institute for Genome Sciences, University of Maryland School of Medicine, Baltimore, MD, USA. ${ }^{3}$ Department of Biophysics, Johns Hopkins University, Baltimore, MD, USA. ${ }^{4}$ Department of Medicine, Duke Human Vaccine Institute, Duke University School of Medicine, Durham, NC, USA. ${ }^{5}$ School of Public Health, University of Maryland College Park, College Park, MD, USA.

Received: 16 February 2021 Accepted: 18 July 2021

Published online: 18 September 2021

\section{References}

1. Herbenick D, Reece M, Sanders SA, Dodge B, Ghassemi A, Fortenberry JD. Women's vibrator use in sexual partnerships: results from a nationally representative survey in the United States. I Sex Marital Ther. 2010;36(1):49-65

2. U.S. Food and Drug Administration. Product Classification. 2021. https:// www.accessdata.fda.gov/scripts/cdrh/cfdocs/cfPCD/classification.cfm? ID=NUC. Accessed 6 September 2021. 
3. Hillier SL, Moench T, Shattock R, Black R, Reichelderfer P, Veronese F. In vitro and in vivo: the story of nonoxynol 9. J Acquir Immune Defic Syndr. 2005;39(1):1-8.

4. Moench TR, Mumper RJ, Hoen TE, Sun M, Cone RA. Microbicide excipients can greatly increase susceptibility to genital herpes transmission in the mouse. BMC Infect Dis. 2010;10:331.

5. Maierhofer C, Rice CE, Wang SH, Fields KS, Ervin M, Turner AN. Lubricant use and rectal chlamydial and gonococcal infections among men who engage in receptive anal intercourse. Sex Transm Dis. 2016:43(7):423-8.

6. Dezzutti CS, Brown ER, Moncla B, Russo J, Cost M, Wang L, Uranker K, Kunjara Na, Ayudhya RP, Pryke K, Pickett J, et al. Is wetter better? An evaluation of over-the-counter personal lubricants for safety and anti-HIV-1 activity. PLoS ONE. 2012;7(11): e48328.

7. Fuchs EJ, Lee LA, Torbenson MS, Parsons TL, Bakshi RP, Guidos AM, Wahl RL, Hendrix CW. Hyperosmolar sexual lubricant causes epithelial damage in the distal colon: potential implication for HIV transmission. J Infect Dis. 2007;195(5):703-10

8. McClelland RS, Richardson BA, Graham SM, Masese LN, Gitau R, Lavreys L, Mandaliya K, Jaoko W, Baeten JM, Ndinya-Achola JO. A prospective study of risk factors for bacterial vaginosis in HIV-1-seronegative African women. Sex Transm Dis. 2008;35(6):617-23.

9. Fashemi B, Delaney ML, Onderdonk AB, Fichorova RN. Effects of feminine hygiene products on the vaginal mucosal biome. Microb Ecol Health Dis. 2013;24:19703. https://doi.org/10.3402/mehd.v24i0.19703.

10. Mitchell C, Manhart LE, Thomas K, Fiedler T, Fredricks DN, Marrazzo J. Behavioral predictors of colonization with Lactobacillus crispatus or Lactobacillus jensenii after treatment for bacterial vaginosis: a cohort study. Infect Dis Obstet Gynecol. 2012;2012: 706540.

11. Laniewski P, Owen KA, Khnanisho M, Brotman RM, Herbst-Kralovetz MM Clinical and personal lubricants impact growth of vaginal Lactobacillus species and colonization of vaginal epithelial cells: an in vitro study. Sex Transm Dis. 2020. https://doi.org/10.1097/OLQ.0000000000001272.

12. van der Veer C, Bruisten SM, van der Helm JJ, de Vries HJ, van Houdt $R$. The cervicovaginal microbiota in women notified for Chlamydia trachomatis infection: a case-control study at the sexually transmitted infection outpatient clinic in Amsterdam, The Netherlands. Clin Infect Dis. 2017:64(1):24-31.

13. Gosmann C, Anahtar MN, Handley SA, Farcasanu M, Abu-Ali G, Bowman BA, Padavattan N, Desai C, Droit L, Moodley A, et al. Lactobacillusdeficient cervicovaginal bacterial communities are associated with increased HIV acquisition in young South African Women. Immunity. 2017:46(1):29-37.

14. Edwards D, Panay N. Treating vulvovaginal atrophy/genitourinary syndrome of menopause: how important is vaginal lubricant and moisturizer composition? Climacteric. 2016;19(2):151-61.

15. Cunha AR, Machado RM, Palmeira-de-Oliveira A, Martinez-de-Oliveira $J$, das Neves J, Palmeira-de-Oliveira R. Characterization of commercially available vaginal lubricants: a safety perspective. Pharmaceutics. 2014;6(3):530-42.

16. Rebe KB, De Swardt G, Berman PA, Struthers H, McIntyre JA. Sexual lubricants in South Africa may potentially disrupt mucosal surfaces and increase HIV transmission risk among men who have sex with men. S Afr Med J. 2013:104(1):49-51.

17. Adriaens E, Remon JP. Mucosal irritation potential of personal lubricants relates to product osmolality as detected by the slug mucosal irritation assay. Sex Transm Dis. 2008;35(5):512-6.

18. Vishwanathan SA, Morris MR, Wolitski RJ, Luo W, Rose CE, Blau DM, Tsegaye T, Zaki SR, Garber DA, Jenkins LT, et al. Rectal application of a highly osmolar personal lubricant in a macaque model induces acute cytotoxicity but does not increase risk of SHIV infection. PLOS ONE. 2015;10(4): e0120021.

19. Ayehunie S, Wang YY, Landry T, Bogojevic S, Cone RA. Hyperosmolal vaginal lubricants markedly reduce epithelial barrier properties in a three-dimensional vaginal epithelium model. Toxicol Rep. 2018:5:134-40.

20. Mitchell C, Marrazzo J. Bacterial vaginosis and the cervicovaginal immune response. Am J Reprod Immunol. 2014;71(6):555-63.

21. Masson L, Arnold KB, Little F, Mlisana K, Lewis DA, Mkhize N, Gamieldien H, Ngcapu S, Johnson L, Lauffenburger DA, et al. Inflammatory cytokine biomarkers to identify women with asymptomatic sexually transmitted infections and bacterial vaginosis who are at high risk of HIV infection. Sex Transm Infect. 2016;92(3):186-93.
22. Arnold KB, Burgener A, Birse K, Romas L, Dunphy LJ, Shahabi K, Abou M, Westmacott GR, McCorrister S, Kwatampora J, et al. Increased levels of inflammatory cytokines in the female reproductive tract are associated with altered expression of proteases, mucosal barrier proteins, and an influx of HIV-susceptible target cells. Mucosal Immunol. 2016;9(1):194-205.

23. Bollen LJ, Blanchard K, Kilmarx PH, Chaikummao S, Connolly C, Wasinrapee P, Srivirojana N, Achalapong J, Tappero JW, McNicholl JM. No increase in cervicovaginal proinflammatory cytokines after Carraguard use in a placebo-controlled randomized clinical trial. J Acquir Immune Defic Syndr. 2008;47(2):253-7.

24. Anderson DJ, Williams DL, Ballagh SA, Barnhart K, Creinin MD, Newman DR, Bowman FP, Politch JA, Duerr AC, Jamieson DJ. Safety analysis of the diaphragm in combination with lubricant or acidifying microbicide gels: effects on markers of inflammation and innate immunity in cervicovaginal fluid. Am J Reprod Immunol. 2009;61(2):121-9.

25. Smith-McCune K, Chen JC, Greenblatt RM, Shanmugasundaram U, Shacklett BL, Hilton JF, Johnson B, Irwin JC, Giudice LC. Unexpected inflammatory effects of intravaginal gels (universal placebo gel and nonoxynol-9) on the upper female reproductive tract: a randomized crossover study. PLoS ONE. 2015;10(7): e0129769.

26. Ravel J, Brotman RM, Gajer P, Ma B, Nandy M, Fadrosh DW, Sakamoto J, Koenig SSK, Fu L, Zhou X, et al. Daily temporal dynamics of vaginal microbiota before, during and after episodes of bacterial vaginosis. Microbiome. 2013. https://doi.org/10.1186/2049-2618-1-29.

27. de Muinck EJ, Trosvik P, Gilfillan GD, Hov JR, Sundaram AYM. A novel ultra high-throughput $16 \mathrm{~S}$ rRNA gene amplicon sequencing library preparation method for the Illumina HiSeq platform. Microbiome. 2017;5(1):68.

28. Holm JB, Humphrys MS, Robinson CK, Settles ML, Ott S, Fu L, Yang H, Gajer P, He X, McComb E, et al. Ultrahigh-throughput multiplexing and sequencing of $>500$-base-pair amplicon regions on the illumina HiSeq 2500 platform. mSystems. 2019. https://doi.org/10.1128/mSystems. 00029-19.

29. Ravel J, Gajer P, Abdo Z, Schneider GM, Koenig SS, McCulle SL, Karlebach S, Gorle R, Russell J, Tacket CO, et al. Vaginal microbiome of reproductiveage women. Proc Natl Acad Sci U S A. 2011;108(Suppl 1):4680-7.

30. France $M$, Bing M, Gajer P, Brown S, Humphrys MS, Holm JB, Waetjen E, Brotman RM, Ravel J. VALENCIA: a nearest centroid classification method for vaginal microbial communities based on composition. Microbiome. 2020;166(8). https://doi.org/10.1186/s40168-020-00934-6.

31. McKinnon LR, Achilles SL, Bradshaw CS, Burgener A, Crucitti T, Fredricks DN, Jaspan HB, Kaul R, Kaushic C, Klatt N, et al. The evolving facets of bacterial vaginosis: implications for HIV transmission. AIDS Res Hum Retrovir. 2019;35(3):219-28.

32. Masson L, Mlisana K, Little F, Werner L, Mkhize NN, Ronacher K, Gamieldien $\mathrm{H}$, Williamson C, McKinnon LR, Walzl G, et al. Defining genital tract cytokine signatures of sexually transmitted infections and bacterial vaginosis in women at high risk of HIV infection: a cross-sectional study. SexTransm Infect. 2014;90(8):580-7.

33 Shannon CE. The mathematical theory of communication. MD Comput 1997. 1963;14(4):306-17.

34. Yue JCCM. A similarity measure based on species proportions. Commun Stat-Theory Methods. 2005:34:2123-31.

35. Arnold IC, Mathisen S, Schulthess J, Danne C, Hegazy AN, Powrie F. $\mathrm{CD} 11 \mathrm{C}(+)$ monocyte/macrophages promote chronic Helicobacter hepaticus-induced intestinal inflammation through the production of IL-23. Mucosal Immunol. 2016:9(2):352-63.

36. Masson L, Passmore JA, Liebenberg LJ, Werner L, Baxter C, Arnold KB, Williamson C, Little F, Mansoor LE, Naranbhai V, et al. Genital inflammation and the risk of HIV acquisition in women. Clin Infect Dis. 2015;61 (2):260-9.

37. Bonecchi R, Sozzani S, Stine JT, Luini W, D'Amico G, Allavena P, Chantry D, Mantovani A. Divergent effects of interleukin-4 and interferon-gamma on macrophage-derived chemokine production: an amplification circuit of polarized T helper 2 responses. Blood. 1998;92(8):2668-71.

38. Nasu K, Sun B, Nishida M, Fukuda J, Narahara H, Miyakawa I. Cultured human endometrial epithelial cells produce thymus and activation-regulated chemokine with stimulation of interleukin-4 and interleukin-13. Fertil Steril. 2004;82(Suppl 3):1014-8.

39. Andrew DP, Chang MS, MCNinch J, Wathen ST, Rihanek M, Tseng J, Spellberg JP, Elias CG 3rd. STCP-1 (MDC) CC chemokine acts specifically on chronically activated Th2 lymphocytes and is produced by 
monocytes on stimulation with Th2 cytokines IL-4 and IL-13. J Immunol. 1998;161(9):5027-38.

40. Wilkinson EM, Laniewski P, Herbst-Kralovetz MM, Brotman RM. Personal and clinical vaginal lubricants: impact on local vaginal microenvironment and implications for epithelial cell host response and barrier function. J Infect Dis. 2019;220(12):2009-18.

41. Hung KJ, Hudson PL, Bergerat A, Hesham H, Choksi N, Mitchell C. Effect of commercial vaginal products on the growth of uropathogenic and commensal vaginal bacteria. Sci Rep. 2020;10(1):7625.

42. Brotman RM. Vaginal microbiome and sexually transmitted infections: an epidemiologic perspective. J Clin Invest. 2011;121(12):4610-7.

43. Rametse CL, Olivier AJ, Masson L, Barnabas S, McKinnon LR, Ngcapu S, Liebenberg $L$, Jaumdally SZ, Gray CM, Jaspan HB, et al. Role of semen in altering the balance between inflammation and tolerance in the female genital tract: does it contribute to HIV risk? Viral Immunol. 2014;27(5):200-6.

44. Sharkey DJ, Macpherson AM, Tremellen KP, Robertson SA. Seminal plasma differentially regulates inflammatory cytokine gene expression in human cervical and vaginal epithelial cells. Mol Hum Reprod. 2007;13(7):491-501.

45. Sharkey DJ, Tremellen KP, Jasper MJ, Gemzell-Danielsson K, Robertson SA. Seminal fluid induces leukocyte recruitment and cytokine and chemokine mRNA expression in the human cervix after coitus. J Immunol. 2012;188(5):2445-54

46. Doherty IA. Sexual networks and sexually transmitted infections: innovations and findings. Curr Opin Infect Dis. 2011;24(1):70-7.

47. Allsworth JE, Peipert JF. Prevalence of bacterial vaginosis: 2001-2004 National Health and Nutrition Examination Survey data. Obstet Gynecol. 2007;109(1):114-20

48. Forney LJ, Gajer P, Williams CJ, Schneider GM, Koenig SS, McCulle SL, Karlebach S, Brotman RM, Davis CC, Ault K, et al. Comparison of selfcollected and physician-collected vaginal swabs for microbiome analysis. J Clin Microbiol. 2010;48(5):1741-8.

49. Bai G, Gajer P, Nandy M, Ma B, Yang H, Sakamoto J, Blanchard MH, Ravel J, Brotman RM. Comparison of storage conditions for human vaginal microbiome studies. PLoS ONE. 2012;7(5): e36934.

50. Srinivasan S, Liu C, Mitchell CM, Fiedler TL, Thomas KK, Agnew KJ, Marrazzo JM, Fredricks DN. Temporal variability of human vaginal bacteria and relationship with bacterial vaginosis. PLoS ONE. 2010;5(4): e10197.

51. Dezzutti CS, Hendrix CW, Marrazzo JM, Pan Z, Wang L, Louissaint N, Kalyoussef S, Torres NM, Hladik F, Parikh U, et al. Performance of swabs, lavage, and diluents to quantify biomarkers of female genital tract soluble mucosal mediators. PLOS ONE. 2011:6(8): e23136.

52. Snowhite IV, Jones WE, Dumestre J, Dunlap K, Braly PS, Hagensee ME. Comparative analysis of methods for collection and measurement of cytokines and immunoglobulins in cervical and vaginal secretions of HIV and HPV infected women. J Immunol Methods. 2002;263(1-2):85-95.

53. Marks MA, Eby Y, Howard R, Gravitt PE. Comparison of normalization methods for measuring immune markers in cervical secretion specimens. J Immunol Methods. 2012;382(1-2):211-5.

54. Graham C, Chooniedass R, Stefura WP, Lotoski L, Lopez P, Befus AD, Becker AB, HayGlass KT. Stability of pro- and anti-inflammatory immune biomarkers for human cohort studies. J Transl Med. 2017;15(1):53.

55. de Jager W, Bourcier K, Rijkers GT, Prakken BJ, Seyfert-Margolis V. Prerequisites for cytokine measurements in clinical trials with multiplex immunoassays. BMC Immunol. 2009;10:52.

56. Rebholz SL, Melchior JT, Welge JA, Remaley AT, Davidson WS, Woollett LA. Effects of multiple freeze/thaw cycles on measurements of potential novel biomarkers associated with adverse pregnancy outcomes. J Clin Lab Med. 2017. https://doi.org/10.16966/2572-9578.107.

57. Lee JE, Kim SY, Shin SY. Effect of repeated freezing and thawing on biomarker stability in plasma and serum samples. Osong Public Health Res Perspect. 2015;6(6):357-62.

58. Huang WY, Kemp TJ, Pfeiffer RM, Pinto LA, Hildesheim A, Purdue MP. Impact of freeze-thaw cycles on circulating inflammation marker measurements. Cytokine. 2017;95:113-7.

59. Flores R, Shi J, Yu G, Ma B, Ravel J, Goedert JJ, Sinha R. Collection media and delayed freezing effects on microbial composition of human stool. Microbiome. 2015;3:33.

60. Sinha R, Chen J, Amir A, Vogtmann E, Shi J, Inman KS, Flores R, Sampson J, Knight R, Chia N. Collecting fecal samples for microbiome analyses in epidemiology studies. Cancer Epidemiol Biomark Prevent. 2016;25(2):407-16.

61. Budding AE, Grasman ME, Eck A, Bogaards JA, Vandenbroucke-Grauls CM, van Bodegraven AA, Savelkoul PH. Rectal swabs for analysis of the intestinal microbiota. PLoS ONE. 2014:9(7): e101344.

62. Carroll IM, Ringel-Kulka T, Siddle JP, Klaenhammer TR, Ringel Y. Characterization of the fecal microbiota using high-throughput sequencing reveals a stable microbial community during storage. PLoS ONE. 2012;7(10): e46953.

63. Marks MA, Viscidi RP, Chang K, Silver M, Burke A, Howard R, Gravitt PE. Differences in the concentration and correlation of cervical immune markers among HPV positive and negative perimenopausal women. Cytokine. 2011;56(3):798-803

\section{Publisher's Note}

Springer Nature remains neutral with regard to jurisdictional claims in published maps and institutional affiliations.
Ready to submit your research? Choose BMC and benefit from:

- fast, convenient online submission

- thorough peer review by experienced researchers in your field

- rapid publication on acceptance

- support for research data, including large and complex data types

- gold Open Access which fosters wider collaboration and increased citations

- maximum visibility for your research: over $100 \mathrm{M}$ website views per year

At BMC, research is always in progress.

Learn more biomedcentral.com/submissions 